\title{
Research and Application Progress of Intelligent Wearable Devices
}

\author{
LIU Feng ${ }^{1,2}$, HAN Jing-Long ${ }^{1, *}$, QI $\mathrm{Ji}^{2}$, ZHANG Yu ${ }^{1}$, YU Jia-Luo ${ }^{2}$, LI Wen-Peng ${ }^{1}$, LIN Dong ${ }^{2}$, \\ CHEN Ling-Xin ${ }^{2}$, LI Bo-Wei ${ }^{2, *}$ \\ ${ }^{1}$ School of Environment and Materials Engineering, Yantai University, Yantai 264005, China \\ ${ }^{2}$ CAS Key Laboratory of Coastal Environmental Processes and Ecological Remediation, Research Center for Coastal Environmental \\ Engineering and Technology, Yantai Institute of Coastal Zone Research, Chinese Academy of Sciences, Yantai 264003, China
}

\begin{abstract}
Intelligent wearable devices have many characteristics such as convenience, intelligence and real-time. Through the detection and analysis of wearable devices and the real-time transmission of data, it can test vital signs and movement and other important information, monitor human body and provide data support for health status. According to the development of wearable devices in recent years, this article summarizes the different ways to wear wearable devices, the common materials for wearable devices and the different sensing modes of wearable devices. Then its applications in physiological and motion detection are also presented. Finally, the challenges and opportunities faced by the wearable devices are analyzed and prospected.
\end{abstract}

Key Words: Wearable technology; Chip sensor; Real-time monitoring; Physiological detection; Review

\section{Introduction}

In recent years, with the rapid development of intelligent wearable devices such as FitBit and Apple Watch, wearable devices have gradually attracted people's attention. At the same time, with the development of micro capacitors, new materials, wireless transmission technology, etc, more technical support has been provided for wearable sensor devices ${ }^{[1,2]}$. At present, wearable devices are mainly used for in vitro detection of vital signs and sports health status. Wearable sensors directly or indirectly contact the human body, and carry out real-time detection and transmission of some indicators such as glucose $\mathrm{e}^{[3]}, \mathrm{pH}$ value ${ }^{[4]}$ and pulse ${ }^{[5]}$. Wearable devices are mainly used for human body monitoring, and also have a small number of applications in other fields. In addition, wearable devices have a lot of detection and analysis on sweat and physical indicators. Most commercial products are wearable devices for physical index detection.
With the in-depth understanding of wearable sensor technology, detection of more information of human body can be realized, and a new generation of wearable devices can be developed soon ${ }^{[6]}$.

This paper summarizes the researches of wearable devices in recent years, including three wearing patterns of wearable devices, commonly used wearable device materials, common wearable devices of different sensing modes, and classifying them according to different applications of wearable devices. Finally, progresses of the wearable devices are summarized and the future development trend is prospected .

\section{Wearable devices}

Wearable device is a kind of portable device that directly puts the sensor device on the body or integrates it into clothes and other wearable items. It combines wearable technology and sensor, changes the traditional mode of sample collection 
and detection, and directly realizes the integration of sample collection and detection.

\subsection{Wearing patterns of wearable devices}

The common wearable devices with different wearable modes are classified into three categories: contact wearable device $^{[7]}$, implanted wearable device ${ }^{[8,9]}$ and wearable sensor with additional device ${ }^{[10,11]}$. Among them, the contact wearable device directly fixes the sensor to the skin surface, the implanted wearable device uses the sensor for transdermal detection, while the wearable sensor with additional device realizes the wearability by connecting the sensor to the fixed device $^{[12,13]}$.

\subsubsection{Contact wearable device}

The direct contact wearable devices are easy, convenient and flexible, with high flexibility and sensitivity. They need the joint support of a variety of technologies in design and manufacturing. The common contact method is to directly fix the device to the skin surface through adhesive or adsorption force ${ }^{[14,15]}$. Sweat is the most easily contacted skin fluid detected by non-invasive wearable devices, which can provide a lot of information related to human physiological status. Therefore, sweat is one of the most targeted biological fluids for developing non-invasive wearable biosensors. For example, $\mathrm{Lu}$ et $a .^{[16]}$ developed a wearable self-powered sweat monitoring system. In the work, the intelligent system further combined with signal transduction, and the wireless transmission technology could conveniently and accurately display sweat information on personal mobile phones and evaluate personal physiological status. Pu et al. ${ }^{[17]}$ prepared a microfluidic chip integrated by a three-electrode sensor for glucose detection, which could continuously monitor glucose within 48 hours. Bandodkar et al. ${ }^{[18]}$ prepared a skin $\mathrm{pH}$ monitoring sensor based on tattoo paper. In addition to monitoring sweat, direct contact wearable devices are often used for pressure and temperature detection ${ }^{[19]}$. Lee et al. ${ }^{[20]}$ made a kind of contact paper-based strain sensor, which could carry out continuous monitoring at a large angle. As shown in Fig.1A, Oh et al. ${ }^{[7]}$ prepared a highly sensitive flexible temperature
A

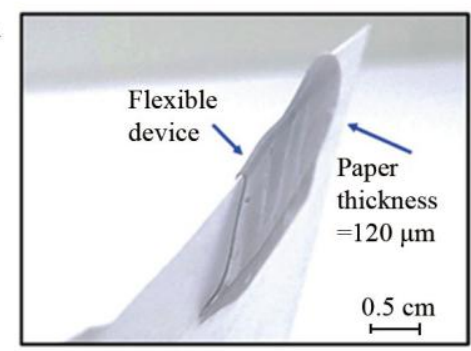

B

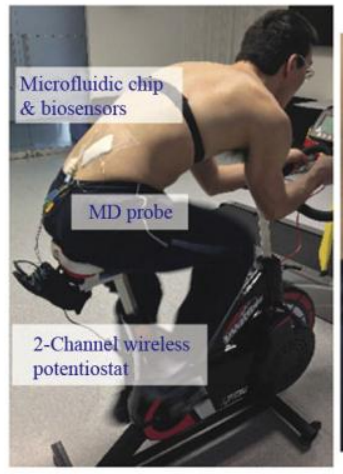

D

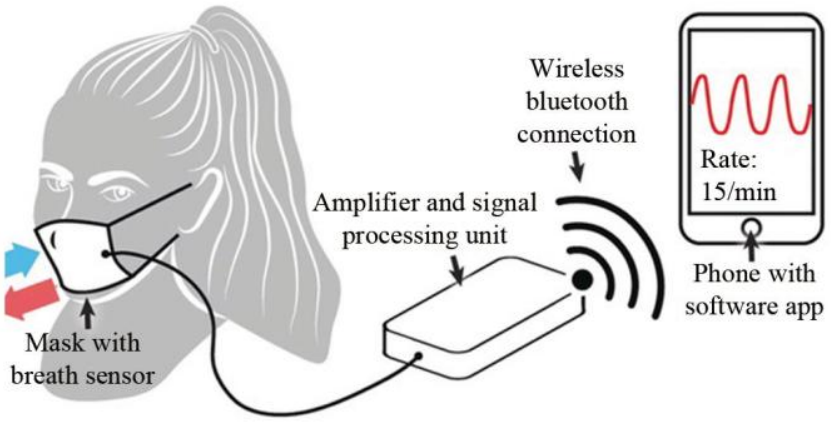

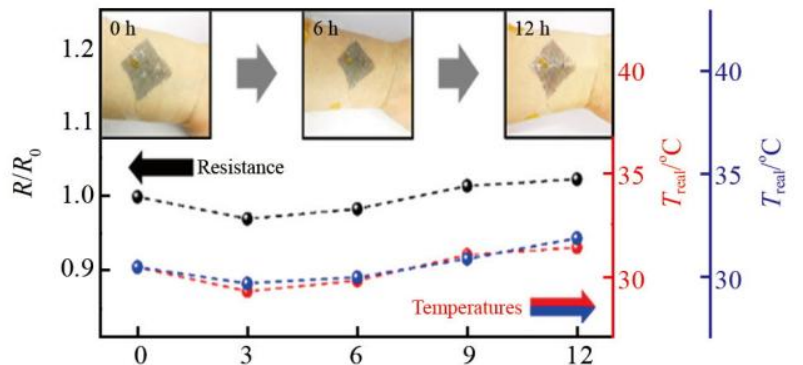

C $\quad t / \mathrm{h}$

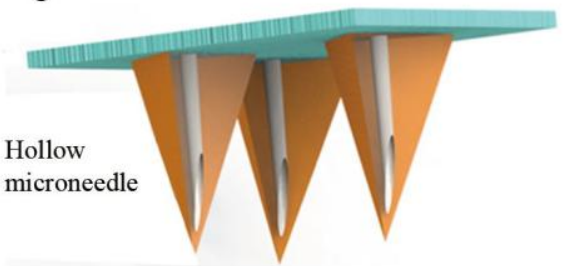

E

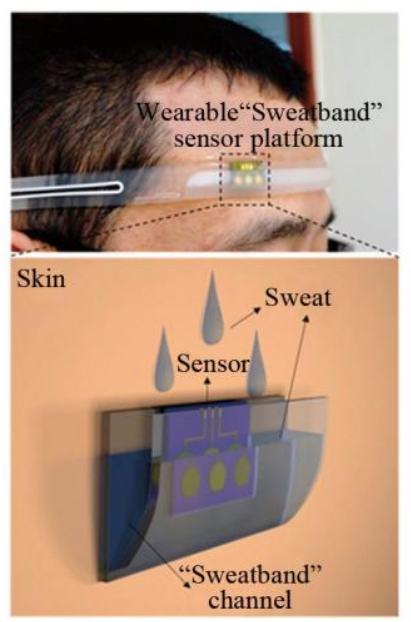

Fig.1 Wearable devices with different ways of wearing: (A) Contact wearable device ${ }^{[7]}$; (B, C) Implanted wearable device ${ }^{[8,9]}$; (D, E) Wearable sensor with additional device ${ }^{[10,11]}$ 
sensor using simulated octopus sucker instead of adhesive. The sensor showed a stable and repeatable detection of skin temperature in the process of repeating adhesion and peeling from the skin, without any skin irritation for a long time.

\subsubsection{Implanted wearable device}

Implantable wearable devices are mainly used for subcutaneous detection in the form of microneedles. Interstitial fluid, as a new source of biomarkers, is of great significance for disease diagnosis. Microneedles provide a minimally invasive method for extracting required molecules from interstitial fluid ${ }^{[21]}$. In recent years, metabolites such as glucose $^{[22]}$, lactic acid ${ }^{[23]}$, alcohol ${ }^{[9]}$ have been detected by interstitial fluid. Compared with blood sampling analysis, microneedle analysis does not cause discomfort and pain, and these effects are more prominent for patients who need to be tested every day ${ }^{[24]}$. Microneedles are a kind of miniaturized traditional hypodermic injection needles with a height of only a few hundred microns, and their size avoids damage to the dermis ${ }^{[25,26]}$. As shown in Fig.1B, Gowers et al. ${ }^{[8]}$ integrated 3D printed microfluidic chip with clinical microdialysis probe approved by FDA, which was connected with a wireless potentiostat manufactured by the laboratory to continuously monitor subcutaneous glucose and lactate levels in real time. As shown in Fig. $1 \mathrm{C}$, Mohan et al. ${ }^{[9]}$ made a microneedle sensor chip for the detection of subcutaneous alcohol. Through the experimental analysis of mouse skin model in vitro, the penetration ability and effectiveness of the biosensor for the monitoring of subcutaneous alcohol concentration were confirmed. Zhao et al. ${ }^{[27]}$ developed an electrochemical biosensor based on microneedles for continuous glucose monitoring. Nightingale et al. ${ }^{[28]}$ prepared a wearable microfluidic sensor for monitoring glucose and lactic acid based on microdialysis probe, which could not only be used for accurate and real-time monitoring, but also reduce the reaction time and make it have rapid clinical response.

\subsubsection{Wearable sensor with additional device}

For wearable sensor with additional device, the sensor itself cannot be directly worn, but can be worn by connecting it to the wearable items, such as smart watch and glasses integrated with audio-visual image processing and recording equipment, wireless connection and sensor, etc. Among them, Google glasses is a typical intelligent wearable sensor with additional device, which has the functions of imaging and video recording and various wireless technologies ${ }^{[29,30]}$. Wearable sensor with additional device is more comfortable when wearing. As shown in Fig.1D, Güder et al. ${ }^{[10]}$ fixed the paper-based sensor into the mask, which was combined with traditional electronic devices to transmit respiratory data to nearby smart phones or tablets for processing, so as to realize real-time monitoring of breathing and breathing. Balakrishnan et $a l .{ }^{[31]}$ also fixed the sensor into the mask, and prepared a wearable device for long-term continuous monitoring of respiratory status. Zhang et al. ${ }^{[32]}$ integrated the sensor into the belt and prepared a wearable breathing sensor based on triboelectric effect. As shown in Fig.1E, Wang et al. ${ }^{[11]}$ developed a wearable sweat sensor to effectively collect and real-time analyze sodium perspiration in indoor sports. The prepared sweat sensor has a good near-Nernstian response to $\mathrm{Na}^{+}$, with a detection limit of $0.8 \times 10^{-6} \mathrm{~mol} / \mathrm{L}$ and good stability. By connecting the sensor device to the wearable device, the integration of more structures and functions can be better realized, such as sensor material ${ }^{[33,34]}$, energy storage device $^{[35,36]}$, near field communication device ${ }^{[37]}$, etc.

\subsection{Common materials for wearable devices}

To meet the requirements of wearable devices, the materials of sensors need to be light, soft and corrosion-resistant. At present, the main materials used in wearable devices include flexible materials ${ }^{[13]}$, paper-based materials ${ }^{[10,31]}$, nanometer materials ${ }^{[38]}$ and organic materials ${ }^{[39]}$.

\subsubsection{Flexible materials}

As common materials for wearable devices, flexible materials mainly include polydimethylsiloxane (PDMS), polyethylene terephthalate (PET), poly (naphthalene ethylene), polyurethane, polyimide and latex. Such materials exhibit good performances in terms of compatibility, bending, mechanical and temperature stability ${ }^{[40,41]}$. Among many flexible materials, PDMS and PET are the most commonly used ones. For example, Xiao et al. ${ }^{[42]}$ developed a wearable glucose colorimetric sensor based on PDMS, which has good flexibility and viscoelasticity. Zhang et al. ${ }^{[43]}$ integrated the relevant microfluidic channels on the PDMS substrate to prepare a sensor chip. By combining intelligent electronics and microfluidic, a small amount of liquid samples could be accurately transmitted to the sensor, and the function of the sensor chip could be fully exerted when the bending curvature radius was $1 \mathrm{~cm}$ and the uniaxial strain was $15 \%$. Zhao et al. ${ }^{[4]}$ coated copper on PET to prepare wearable devices for monitoring of human respiration. As shown in Fig.2A, Nie et $a l .^{[45]}$ prepared a flexible microfluidic chip for continuous detection of sweat for a long time. The chip was made of three layers of PET film. During detection, the chip on the skin surface absorbed the liquid on the body surface through a filter integrated in the inlet, and then entered into the microchannel and the sensing cavity for detection through capillarity. The ultra light weight, high fluidity, scalability, low cost and compatibility of flexible materials made it possible to integrate many other components ${ }^{[46,47]}$. The flexibility provided by scalability can not only relieve the mechanical strain 

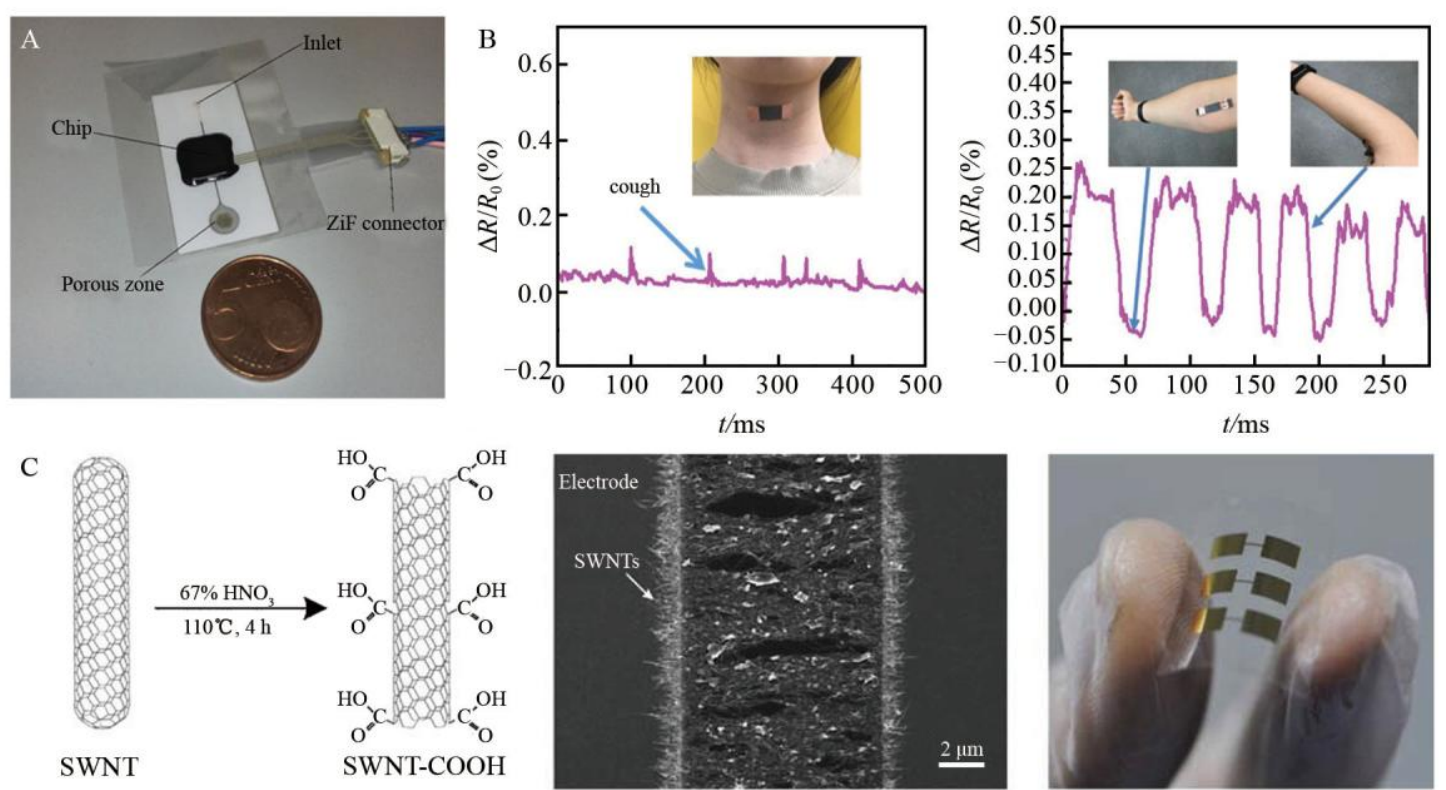

SWNT

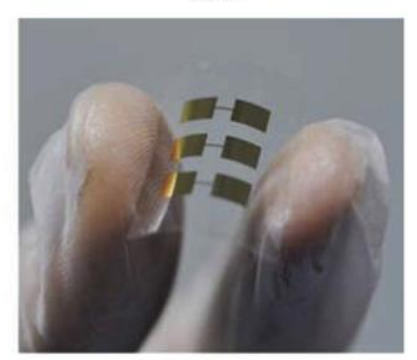

D
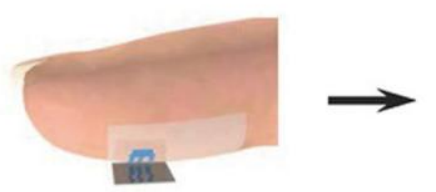

Transfer OPT

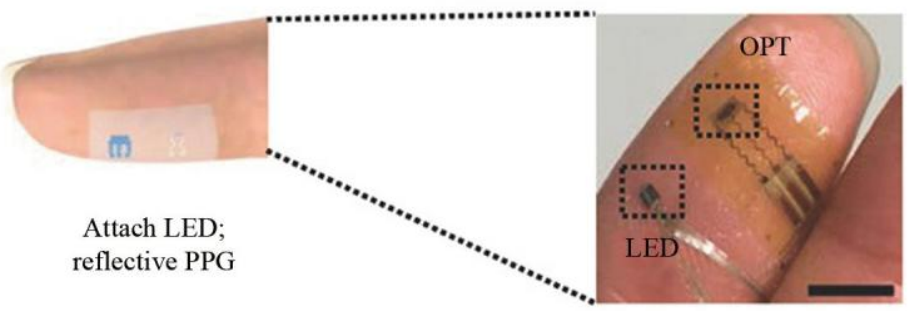

Fig.2 Common materials for wearable devices: (A) Flexible material ${ }^{[45]}$; (B) Paper-based material ${ }^{[51]}$; (C) Nanometer material ${ }^{[52]}$; (D) Organic material ${ }^{[53]}$

caused by bending, but also relieve the mechanical strain caused by other disturbances such as compression, tension and torsion $^{[48]}$. The flexible and stretchable wearable device can be firmly combined with the skin, reduce the stress at the interface as much as possible, and realize the monitoring of human activities and personal health under the strong pressure generated in the process of normal body movement and muscle movement ${ }^{[49,50]}$.

\subsubsection{Paper-based materials}

Owing to its unique characteristics, paper-based device has many advantages such as easy recycling, low cost, strong water absorption and flexibility ${ }^{[54-59]}$. The unique characteristics of paper-based wearable devices enable them to be well used in respiratory monitoring. The wearable paper-based moisture sensor prepared by using the paper-based good water absorption can detect the change of moisture caused by respiration, so as to detect personal respiration. Compared with traditional devices, the cost of paper-based devices are very low ${ }^{[10,31]}$. The integration of paper-based materials with other functional materials can give full play to its advantages, such as metal nanowires ${ }^{[60]}$, graphene $^{[51,61]}$, nanoparticles ${ }^{[20]}$ and zinc oxide nanowires ${ }^{[62]}$. Wang et $a l .{ }^{[61]}$ proposed a new flexible electrochemical sweat detection platform based on graphene paper. In this work, copper submicron buds were deposited on graphene paper with $\mathrm{MoS}_{2}$ nanocrystals. Glucose and lactic acid in sweat were quantitatively analyzed by amperometric $\mathrm{i}-\mathrm{t}$ method. As shown in Fig.2B, Qi et al. ${ }^{[51]}$ used Meyer-rod coating process to coat graphene dispersion on mulberry paper to prepare mulberry paper cornerstone graphene strain sensor. The strain sensor had a mechanical resistance of $1.13 \mathrm{MPa}$, a gauge factor of 3.82 and durability of 1000 bending tests. When the wearer coughed and bent his elbow, the sensor also made corresponding response. By combining paper with other materials, the strength of paper-based equipment can be improved. For example, by combining cotton thread and filter paper, Xiao et al. ${ }^{[63]}$ designed and prepared a wearable device for glucose detection based on microfluidic paper-based chip, which provided a new idea for the development of paper-based wearable devices.

\subsubsection{Nanometer materials}

Common nanomaterials for wearable devices include carbon nanotubes ${ }^{[64]}$, metal nanowires ${ }^{[65]}$, metal oxide nanowires ${ }^{[38]}$ and conductive polymer nanowires ${ }^{[66]}$. Yamada et al. ${ }^{[64]}$ prepared wearable devices using single-walled carbon nanotube films for human motion detection. As shown in 
Fig.2C, Liu et al. ${ }^{[52]}$ made a $\mathrm{pH}$ sensor based on the functionalized single-walled carbon nanotubes with carboxyl groups in dielectrophoresis. The carboxyl group modified by the sensor reacted with $\mathrm{H}^{+}$and $\mathrm{OH}^{-}$, realizing the detecting of the $\mathrm{pH}$ value. Many technologies have been developed to prepare carbon nanotubes with different morphologies, including vertically grown carbon nanotubes ${ }^{[67]}$, buckled and aligned carbon nanotubes ${ }^{[68]}$ and suspended carbon nanotubes ${ }^{[64]}$. For metal nanomaterials, there are mainly gold nanowires, silver nanowires and copper nanowires. Mao et $a l{ }^{[65]}$ developed a pressure sensor combining silver nanowires and PDMS to monitor the movements such as touching, swallowing, bending and twisting. Metal oxide nanowires have attracted extensive attention in wearable energy devices due to their layered structure, high electron mobility and unique piezoelectric properties ${ }^{[67]}$. $\mathrm{ZnO}$ and $\mathrm{ZnS}$ nanowire materials are often used in the manufacture of wearable sensors ${ }^{[69]}$. For example, Arrabito et al. ${ }^{[38]}$ prepared a flexible wearable sensor using nano $\mathrm{ZnO}$ to detect pulse. Zhao et al. ${ }^{[70]}$ developed a strain sensor chip using complementary-metaloxide-semiconductor technology to detect strain. This sensor showed high sensitivity and ultra-thin structure.

\subsubsection{Organic materials}

Organic sensors based on organic semiconductors or conductive materials have become one of the commonly used materials for wearable devices due to their flexibility, extensibility, low cost and light weight. The unique advantage of organic semiconductors and conductive materials is that their electrical, mechanical, chemical and optical properties can be optimized by reasonable molecular design ${ }^{[71,72]}$. The adjustable optical range of organic semiconductor optical sensors can achieve the absorption wavelength from near-infrared to ultraviolet region through molecular design. Therefore, these sensors are widely used for continuous and real-time monitoring of individual physiological state ${ }^{[73,74]}$. Yokota et al. ${ }^{[73]}$ developed a wearable electronic skin device based on polymer light-emitting diodes and organic photodetectors to detect pulse and blood oxygen levels. Lee et $a l{ }^{[75]}$ developed a reflective organic pulse oxygen sensor with ultra-low power consumption. The proposed monolithic organic pulse oxygen sensor could be operated at $24 \mu \mathrm{W}$ electrical power. As shown in Fig.2D, Xu et al. ${ }^{[53]}$ developed a photoelectric volume sensor based on high-sensitivity organic phototransistor. The ultra-thin wearable device could continuously monitor the change of heart rate and accurately tracked the change of pulse pressure of various parts of the body. Khan et al. ${ }^{[76]}$ prepared a two-dimensional reflective oxygen saturation sensor. The array realized two-dimensional mapping of oxidation in a region, instead of the one-dimensional mapping in the previous study. Park et al. ${ }^{[77]}$ made use of organic photodiodes to manufacture a near-infrared response photoelectric volume sensor. The ultra-thin photoelectric sensor still had good stability after more than $10^{3}$ bending cycles under severe mechanical deformation with bending radius less than $3 \mu \mathrm{m}$.

\subsection{Wearable devices with different sensing modes}

How to realize the signal conversion of wearable devices is the key to the normal operation of sensors. According to different sensing methods, wearable devices can be divided into three categories, namely chemical sensors ${ }^{[78]}$, optical sensors $^{[73]}$ and electromechanical sensors ${ }^{[79]}$.

\subsubsection{Wearable chemical sensors}

Wearable chemical sensors are mainly detected by converting chemical signals into optical signals or electrical signals. The commonly used detection method for chemical to optical signals is colorimetry, while the chemical to electrical signals are mainly detected by electrochemical ${ }^{[78,80]}$. The main advantages of colorimetry are ultra-low cost, simple equipment, and no need of external electronic equipment and detection device ${ }^{[81]}$. Koh et $a l^{[82]}$ developed a wearable colorimetric sweat detection sensor to detect sweating rate, sweating amount, $\mathrm{pH}$ value, chloride and lactic acid concentration. As shown in Fig.3A, Curto et al. ${ }^{[78]}$ produced a wearable chip sensor based on ionic gels and microfluidics. Compared with standard color chart, the $\mathrm{pH}$ value of sweat could be obtained by observing the color changes. Many wearable chemical sensors are electrical signals in nature. Once they come into contact with biological fluids, they start to work. Meanwhile, many electrochemical sensors can work continuously and have reversibility ${ }^{[83]}$. As shown in Fig.3B, Gao et $a l .{ }^{[80]}$ developed a sweat detection sensor based on electrochemistry, which could simultaneously measure sweat metabolites, electrolytes and skin temperature. Zhang et al. ${ }^{[84]}$ developed a chip sensor for detecting sweat. The chip sensor had good salt sensitivity. With the increase of salt concentration, the current of the chip sensor decreased. The sweat sensor could real-time monitor the sweat of human body during indoor movement by sticking the chip on the human arm. Matzeu et al. ${ }^{[85]}$ prepared a microfluidic chip for detecting $\mathrm{Na}^{+}$content in sweat, which combined solid-contact ion-selective electrode and liquid-junction-free reference electrode together to realize real-time monitoring through wireless electronic platform. For wearable chemical sensing devices, it is a challenge to extract and enrich targets in sweat due to that the target analytes such as ions, molecules and proteins have low content in body fluids and are difficult to extract ${ }^{[86,87]}$.

\subsubsection{Wearable optical sensors}

Wearable optical sensors introduce light into the body, and 

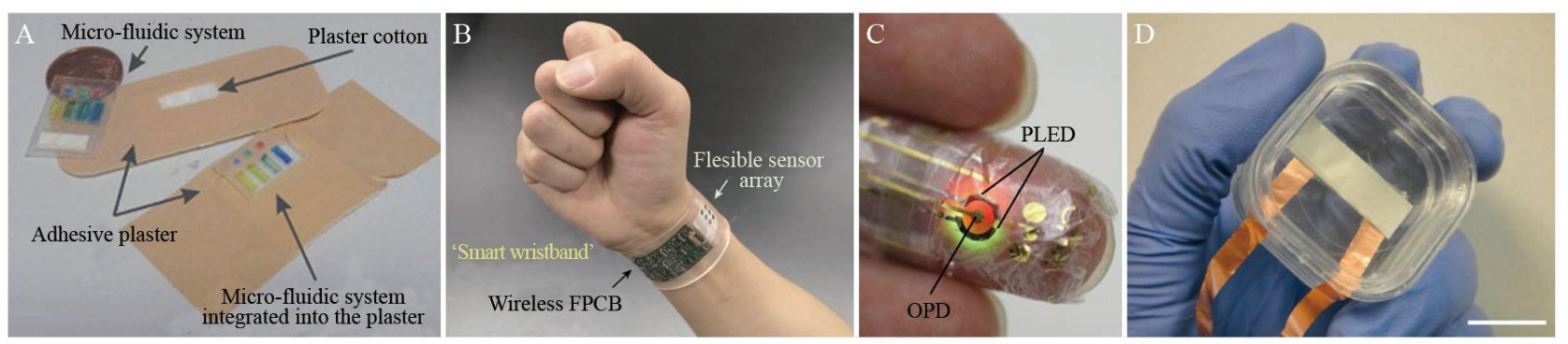

Fig.3 Wearable devices with different sensing modes: $(\mathrm{A}, \mathrm{B})$ Wearable chemical sensors ${ }^{[78,80]}$; (C) Wearable optical sensors ${ }^{[73]}$; (D) Wearable electromechanical sensors ${ }^{[79]}$

display human body information on the optical detector by using the changes of light scattering or light absorption. Advanced miniaturized photoelectric devices, combined with wearable substrates, can perform various sensing functions on the skin surface ${ }^{[73,80]}$. Photodetectors and light-emitting diodes of different colors are integrated together and interconnected with external circuits. The light signal penetrates the skin, and the tissue oxygen level is monitored in real time according to the difference of spectral response between oxyhemoglobin and deoxyhemoglobin ${ }^{[88]}$. As shown in Fig. 3C, Yokota et al. ${ }^{[73]}$ developed a super flexible reflective pulse oxygen sensor. The total thickness of the ultra-thin sensor was only $3 \mu \mathrm{m}$, and the visual display could directly display the number and color. Lochner et al. ${ }^{[89]}$ prepared a pulse oxygen sensor based on organic materials. The accuracy of the sensor was very high, and the measurement error of pulse rate and blood oxygen was $1 \%$ and $2 \%$, respectively. Kim et al. ${ }^{[90]}$ used quantum dots as active materials to fabricate ultra-thin photodiodes, which were connected to the front and back of fingertips to realize the measurement of transmission pulse oxygen saturation. In addition to conventional pulse oxygen detection, photoelectric sensors can also detect muscle movement. Bansal et al. ${ }^{[91]}$ prepared a wearable sensor by combining organic light-emitting diodes with organic photodiodes. The wearable sensor was composed of four photodiodes around a central light source, which could measure and distinguish muscle stretching and contraction while detecting blood oxygen, so as to control the movement of the manipulator. Wearable sensors based on optical sensing can be well applied to non-invasive detection. Through further research on the sensing system and optical data, a more comprehensive detection of human health can be achieved ${ }^{[92]}$.

\subsubsection{Wearable electromechanical sensors}

Wearable electromechanical sensors mainly monitor body signals by detecting the changes in body surface resistance, capacitance or conductivity. Common wearable electromechanical sensors include temperature sensors, strain sensors and pressure sensors ${ }^{[87,93]}$.

It is of great significance for continuous and real-time monitoring of body temperature. Pan et al. ${ }^{[94]}$ prepared a flexible temperature sensor, in which the high transparent temperature sensor was well connected with the display device. The temperature sensor based on carbon nanomaterials can increase the thermal response range and improve the sensitivity of the sensing device, and its resistance is strictly dependent on temperature ${ }^{[95,96]}$. Giuliani et al ${ }^{[96]}$ developed a skin temperature sensor using multi walled carbon nanotubes. The resistance sensitivity to temperature was close to -0.004 $\mathrm{K}^{-1}$, and its absolute value was equivalent to the highest value in metals. Yang et al. ${ }^{[97]}$ prepared a super sensitive wearable temperature sensor based on graphene nanowall and PDMS to monitor body temperature in real time, showing relatively fast response recovery and long-term stability.

Strain sensors play an important role in motion monitoring. The sensitivity (gauge factor) of most metal-based strain equipment is $2-5$, the sensitivity based on semiconductor is 100 or more, and the sensitivity based on flexible materials is between 1 and 100. In addition, the sensitivity also depends on the sensing mechanism, material structure and other factors $^{[98-100]}$. Souri et al. ${ }^{[101]}$ used graphene nanoplates and carbon black to prepare strain sensors for detecting human motion, which could identify the movements of fingers, wrists and knees. Wang et al. ${ }^{[102]}$ prepared a wearable strain sensor using graphene-based fabric, which could detect some weak human movements with high sensitivity. Lee et al. ${ }^{[20]}$ made a paper-based strain sensor, of which the gauge factor was 41.98 in tensile strain test and 21.36 in compression strain test, showing good sensitivity.

Pressure sensor is the most common electromechanical sensor, which usually converts the applied force into electrical signal output. Gong et al. ${ }^{[60]}$ built a highly sensitive wearable piezoresistive pressure sensor on the basis of PDMS. The pressure sensitivity of piezoresistive pressure sensor is high, but the problem is that most of them need external power to maintain continuous monitoring. The piezoelectric sensor based on piezoelectric effect produces charge change when subjected to mechanical stress. The high sensitivity and rapid response of piezoelectric sensor make it have great advantages and development potential in the application of wearable sensor. As shown in Fig.3D, Persano et al. ${ }^{[79]}$ prepared a 
flexible pressure sensor with ultra-high sensitivity. Under bending conditions, the sensor showed a high sensitivity, i.e., a current of $40 \mathrm{nA}$ and a voltage of about $1.5 \mathrm{~V}$.

The capacitance of the capacitive sensor changes under the action of mechanical stimulation, thus generating a signal. $\mathrm{Li}$ et al. ${ }^{[103]}$ reported a soft-matter interdigitated capacitor sensor. By integrating the interdigital capacitor with the capacitance sensor chip, it could capture the respiratory frequency caused by human chest movement. Compared with other types of wearable sensor, wearable capacitive sensor has high sensitivity and low power consumption.

As a new sensing mechanism of iontronic interface, the ionophore sensing has a significant improvement in sensitivity and signal-to-noise ratio. Nie et al. ${ }^{[104]}$ developed a flexible tactile sensor to monitor the dynamic pressure wave in cardiovascular circulation using iontronic microdroplet array. Subsequently, the team developed an interface pressure monitoring sensor for diagnosis of chronic venous disease using capacitive iontronic sensor technology ${ }^{[105]}$.
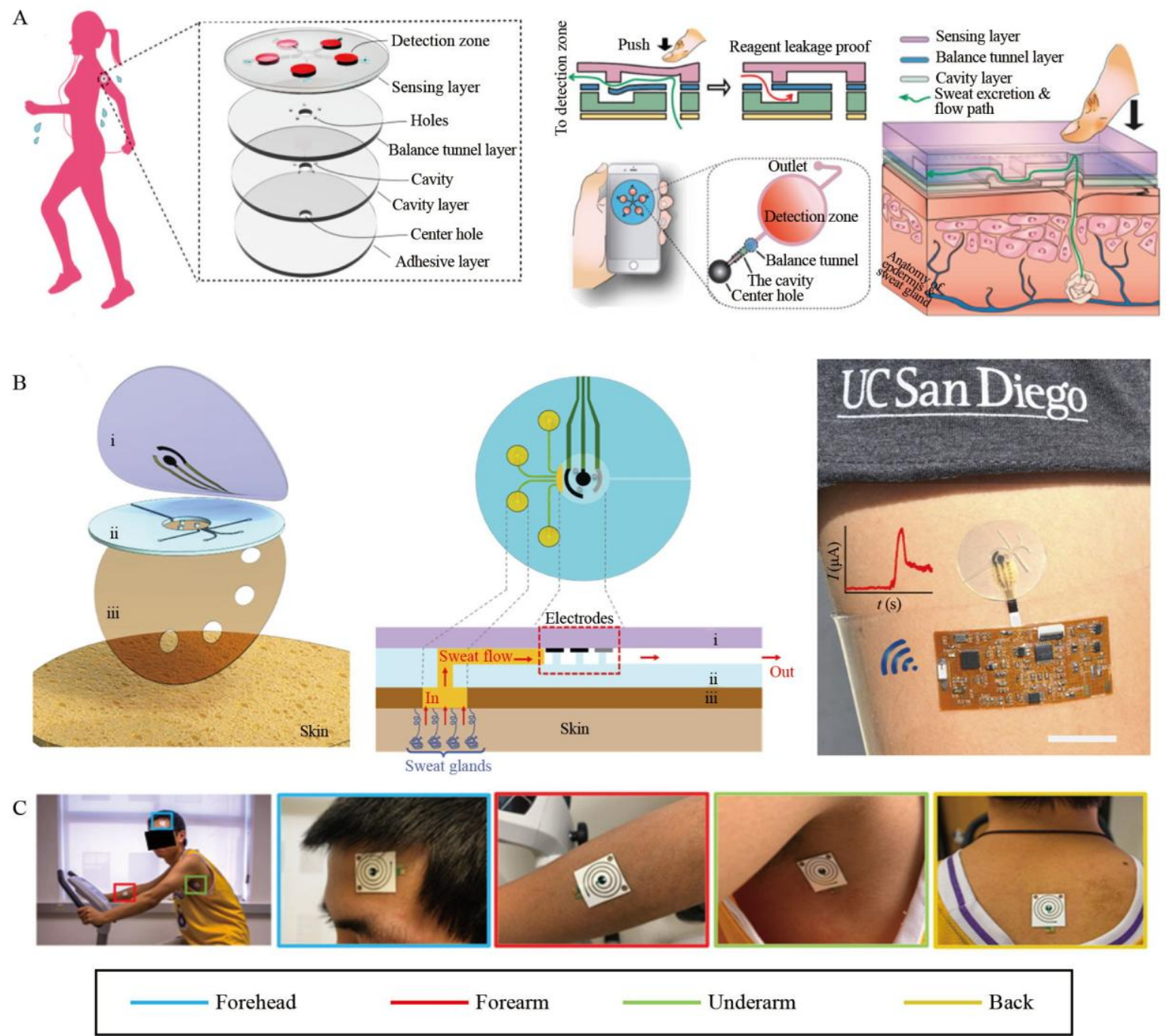

Fig.4 Wearable devices for sweat detection: (A) Structure and function diagram of wearable sensor for glucose detection ${ }^{[42]}$; (B) A microfluidic wearable sensor for glucose and lactate detection ${ }^{[109]} ;(\mathrm{C})$ Wearable sensor for sweat detection ${ }^{[11]}$ 
a wearable colorimetric sensor based on microfluidic chip to detect sweat glucose. The device had five channels, which could detect five times at the same time, and thus could display the slight difference between the glucose concentration during fasting and after oral glucose. Liu et $a l^{[108]}$ proposed a kind of wearable biosensor for high-sensitivity glucose detection. After functionalizing the electrode, the detection range of the glucose sensor was widened to at least 5 orders of magnitude, and the detection limit was as low as $10 \mathrm{nmol} / \mathrm{L}$. As shown in Fig.4B, Martín et $a l^{[109]}$ prepared an epidermal microfluidic detection chip, which combined photolithography technology and screen printing technology, and integrated with a miniaturized flexible electronic board to transmit data wirelessly to mobile devices for sweat sampling and real-time continuous monitoring of glucose and lactic acid. Lee et al. ${ }^{[110]}$ developed a kind of sweat glucose monitoring device integrated by microneedles, which could real-time correct the sweat glucose measurement based on $\mathrm{pH}$ value, temperature and humidity sensing.

The $\mathrm{pH}$ value of sweat reflects the sweating condition and health status of human body. The detection of $\mathrm{pH}$ value of sweat can directly show the dehydration status and physical condition of human body. Caldara et al. ${ }^{[4]}$ assembled the cotton fabric treated with organic modified silicate and small electronic devices with wireless interface together to make a wearable $\mathrm{pH}$ sensor. Saravanakumar et al. ${ }^{[12]}$ prepared a wearable $\mathrm{pH}$ sensor based on $\mathrm{ZnO}$ nanowires and piezoelectric polymers. Huang et al. ${ }^{[113]}$ developed a wearable wireless sensor to detect the $\mathrm{pH}$ value of sweat through dielectric detection and colorimetric measurement. Bandodkar et $a l .^{[18]}$ combined commercially available tattoo paper with traditional screen printing and solid-contact ion-selective electrode to prepare a sensor for monitoring skin $\mathrm{pH}$, which was used for real-time monitoring of human sweating. The potential sensor combined with tattoo paper showed rapid and sensitive response to $\mathrm{pH}$ change. In addition, tattoo paper could withstand mechanical deformation, which met the key requirement of wearable device and could be used on almost any exposed skin surface, and yet beautiful.

The changes of $\mathrm{Na}^{+}$and $\mathrm{K}^{+}$in human sweat are related to the loss of electrolytes. Electrolyte disorder may lead to different body injuries. Garcia-Cordero et al. ${ }^{[114]}$ developed a whole chip integrated wearable sweat sensing system. In the work, a small amount of collected sweat was transported to the chip through capillary action for the detection of $\mathrm{pH}$ value, $\mathrm{Na}^{+}$and $\mathrm{K}^{+}$concentration. Rose et al. ${ }^{[115]}$ prepared a kind of adhesive sensor bandage directly contact with human body, and integrated the sensor on flexible materials to realize the detection of ions in sweat. Cazalé et al. ${ }^{[116]}$ developed sodium perspiration detection sensor based on ion-sensitive electrodes and ion-sensitive field effect transistors, showing good sensitivity.
Sweating rate reflects human health, and a lot of sweating may lead to disorder of the body, dehydration, severe heat stroke and other consequences. As shown in Fig.4C, Nyein et $a .^{[111]}$ introduced a microfluidic sensor patch prepared by roll-to-roll processes to collect sweat and measure the changes of sweat rate, $\mathrm{Na}^{+}, \mathrm{K}^{+}$and glucose in sweat in real time. Matzeu et al. $^{[117]}$ prepared wearable devices by combining with the sweat sampling devices that commonly used in clinical practice, by which the sweat flow was detected by analyzing captured images. Brueck et al.$^{[118]}$ developed a flow detection system based on microfluidic control, which integrated sensors of wireless devices together and could send sweat rate to mobile phones or cloud in real time.

\subsection{Respiratory detection}

As a physiological parameter of human body, respiration is an important basis for judging human health. At present, various special monitors are mainly used for monitoring ${ }^{[119,120]}$ Boland et $a l .{ }^{[121]}$ prepared a conductive composite material made of graphene filled rubber, which had high strain sensitivity, and the working strain exceeded $800 \%$. Atalay et $a l .^{[122]}$ developed a fabric-based strain sensor. The prepared breathing band could be tied around the chest or abdomen to monitor the respiratory rate. Zhao et al. ${ }^{[44]}$ prepared a wearable chest strap, which could not only monitor the respiratory rate, but also detect the change of breathing mode. In particular, the paper-based wearable breath detection is particularly used in the respiratory detection. For example, Güder et al. ${ }^{[10]}$ developed a paper-based moisture sensor on the basis of the water absorption capacity of the paper. In the work, the humidity change caused by respiration was converted into electrical signals, which were used for the monitoring of the breathing mode and respiration rate. In the process of respiration, the change of humidity would result in the corresponding change of the conductivity of the sensor. By monitoring the change of the electrical signal, the respiration was monitored. The paper-based electrical breathing sensor had low cost and simple operation. Balakrishnan et al. ${ }^{[31]}$ prepared a breath monitoring sensor with an optimal sensitivity of $0.0564 \%$ in the linear range, which made it easy to distinguish different breathing methods.

\subsection{Heart rate and blood oxygen detection}

The monitoring of heart rate and blood oxygen monitoring is an important means to ensure the cardiovascular system and even the whole body health. Since the pulse is the direct reflection of heart rate, we think they are the same ${ }^{[123,124]}$. Pang et $a l^{[5]}$ prepared a wearable pressure sensor for amplifying signal and detecting pulse. Schwartz et al. ${ }^{[125]}$ developed a flexible pressure-sensitive organic thin film transistor sensor with a maximum sensitivity of $8.4 \mathrm{kPa}^{-1}$, a response time of 
less than $10 \mathrm{~ms}$, and a high stability of more than 15000 cycles. The wearable sensor prepared by the sensor can be used for noninvasive, high accuracy and continuous monitoring of radial artery pulse. In addition to using pressure to measure heart rate, light-emitting diodes and photodiodes are usually combined, and photoplethysmography is used to simultaneously measure heart rate and blood oxygen ${ }^{[92,126]}$. Haahr et al. ${ }^{[127]}$ developed a low-power wearable device for measurement of reflected pulse oxygen saturation, which was used for blood oxygen monitoring and pulse measurement. Chacon et al. ${ }^{[128]}$ made a wearable device for the continuous measurement of pulse oxygen saturation and heart rate by reflective probe. In the work, the wireless transmission and signal processing technology were used to send measurement signals to mobile devices for real-time signal processing. The accuracy of blood oxygen measurement was at least $98 \%$, and the accuracy rate of heart rate was more than $97 \%$.

\section{Conclusions and prospect}

With the development of various technologies, wearable devices, as the product of the comprehensive application of materials, chemistry, biology, physics and other disciplines, has benefited a lot and developed rapidly. However, there are still some challenges to be solved and there is a huge space for development. First of all, due to the problem that sweat detection equipment cannot be reused, adding a drainage system can realize the recycling of equipment. Secondly, wearable devices usually consume a lot of power to detect and record the transmitted information. Although most devices can carry out continuous detection ranging from several minutes to several hours, they still cannot meet the long-term wearing demand. Therefore, better combination of super capacitor and other capacitive devices can realize smaller and more lasting power supply. Moreover, wearable devices integrate many components, which affect the comfort more or less when wearing. In addition, the biocompatibility of wearable devices also needs to be considered. With the development of wireless transmission and new materials, it can enhance the intelligence and reduce the size of the device to make it more comfortable to wear. Furthermore, combined with the natural biocompatibility and biodegradability of biomaterials, it can be solved its security problem. Finally, the detection range of wearable devices is mainly focused on the detection of physiological health. With the development of materials science, nanotechnology, communication technology and biotechnology, it is also important to synthesize novel sensors to expand the detection range of wearable devices.

In conclusion, although there are still some issues in wearable devices, with the further expansion of synthetic materials and detection methods, and the continuous progress of detection platform and transmission technology, wearable devices will have a broader development prospect.

\section{Conflict of interests}

The authors declare no competing financial interest.

\section{References}

[1] Zhang C F, Kremer M P, Seral-Ascaso A, Park S H, McEvoy N, Anasori B, Gogotsi Y, Nicolosi V. Adv. Funct. Mater., 2018, 28(9): 1705506

[2] Jiang Q, Wu C S, Wang Z J, Wang A C, He J H, Wang Z L, Alshareef H N. Nano Energy, 2018, 45: 266-272

[3] Lee H, Choi T K, Lee Y B, Cho H R, Ghaffari R, Wang L, Choi H J, Chung T D, Lu N S, Hyeon T, Choi S H, Kim D H. Nat. Nanotechnol., 2016, 11(6): 566

[4] Caldara M, Colleoni C, Guido E, Re V, Rosace G. Sens. Actuators B, 2016, 222: 213-220

[5] Pang C, Koo J H, Nguyen A, Caves J M, Kim M G, Chortos A, Kim K, Wang P J, Tok J B H, Bao Z A. Adv. Mater., 2015, 27(4): 634-640

[6] Choi J, Ghaffari R, Baker L B, Rogers J A. Sci. Adv., 2018, 4(2): eaar3921

[7] Oh J H, Hong S Y, Park H, Jin S W, Jeong Y R, Oh S Y, Yun J, Lee H, Kim J W, Ha J S. ACS Appl. Mater. Interfaces, 2018, 10(8): 7263-7270

[8] Gowers S A N, Curto V F, Seneci C A, Wang C, Anastasova S, Vadgama P, Yang G Z, Boutelle M G. Anal. Chem., 2015, 87(15): 7763-7770

[9] Mohan A M V, Windmiller J R, Mishra R K, Wang J. Biosens. Bioelectron., 2017, 91: 574-579

[10] Güder F, Ainla A, Redston J, Mosadegh B, Glavan A, Martin T J, Whitesides G M. Angew. Chem. Int. Ed., 2016, 55(19): $5727-5732$

[11] Wang S Q, Wu Y J, Gu Y, Li T, Luo H, Li L H, Bai Y Y, Li L L, Liu L, Cao Y D, Ding H Y, Zhang T. Anal. Chem., 2017, 89(19): 10224-10231

[12] Amjadi M, Kyung K U, Park I, Sitti M. Adv. Funct. Mater., 2016, 26(11): 1678-1698

[13] Tran Quang T, Lee N E. Adv. Mater., 2016, 28(22): 4338-4372

[14] Wang S, Chinnasamy T, Lifson M A, Inci F, Demirci U. Trends Biotechnol., 2016, 34(11): 909-921

[15] Liao X, Zhang Z, Liao Q, Liang Q, Ou Y, Xu M, Li M, Zhang G, Zhang Y. Nanoscale, 2016, 8(26): 13025-13032

[16] Lu Y, Jiang K, Chen D, Shen G. Nano Energy, 2019, 58: 624-632

[17] Pu Z H, Zou C W, Wang R D, Lai X C, Yu H X, Xu K X, Li D C. Biomicrofluidics, 2016, 10(1): 011910

[18] Bandodkar A J, Hung V W S, Jia W Z, Valdes-Ramirez G, Windmiller J R, Martinez A G, Ramirez J, Chan G, Kerman K, Wang J. Analyst, 2013, 138(1): 123-128

[19] Chen J X, Wen H J, Zhang G L, Lei F, Feng Q, Liu Y, Cao X D, Bong H. ACS Appl. Mater. Interfaces, 2020, 12(6): 7565-7574

[20] Lee D J, Kim D Y. IEEE Access, 2019, 7: 77200-77207 
[21] Dervisevic M, Alba M, Prieto-Simon B, Voelcker N H. Nano Today, 2020, 30: 100828

[22] Lee S J, Yoon H S, Xuan X, Park J Y, Paik S J, Allen M G. Sens. Actuators B, 2016, 222: 1144-1151

[23] Calio A, Dardano P, Di Palma V, Bevilacqua M F, Di Matteo A, Iuele H, De Stefano L. Sens. Actuators B, 2016, 236: 343-349

[24] Kinnamon D, Ghanta R, Lin K C, Muthukumar S, Prasad S. Sci Rep, 2017, 7: 13312

[25] Larraneta E, Lutton R E M, Woolfson A D, Donnelly R F. Mater. Sci. Eng. R., 2016, 104: 1-32

[26] Ventrelli L, Strambini L M, Barillaro G. Adv. Healthc. Mater., 2015, 4(17): 2606-2640

[27] Zhao L, Wen Z Z, Jiang F J, Zheng Z Z, Lu S Z. RSC Adv., 2020, 10(11): 6163-6171

[28] Nightingale A M, Leong C L, Burnish R A, Hassan S U, Zhang Y, Clough G F, Boutelle M G, Voegeli D, Niu X Z. Nat. Commun., 2019, 10: 2741

[29] Feng S, Caire R, Cortazar B, Turan M, Wong A, Ozcan A. ACS Nano, 2014, 8(3): 3069-3079

[30] Roggen D, Magnenat S, Waibel M, Troster G. IEEE Robot. Autom. Mag., 2011, 18(2): 83-95

[31] Balakrishnan V, Dinh T, Foisal A M, Nguyen T, Phan H P, Dao D V, Nguyen N T. IEEE Sens. J., 2019, 19(24): 11784-11790

[32] Zhang H, Zhang J, Hu Z, Quan L, Shi L, Chen J, Xuan W, Zhang Z, Dong S, Luo J. Nano Energy, 2019, 59: 75-83

[33] Fan Y-Q, Gao F, Wang M, Zhuang J, Tang G, Zhang Y-J. Chinese J. Anal. Chem., 2017, 45(3): 455-462

[34] Qian X, Su M, Li F, Song Y. Acta Chim. Sin., 2016, 74(7): $565-575$

[35] Jia R, Shen G, Qu F, Chen D. Energy Storage Materials, 2020, 27: $169-186$

[36] Sumboja A, Liu J, Zheng W G, Zong Y, Zhang H, Liu Z. Chemical Society Reviews, 2018, 47(15): 5919-5945

[37] Cao Z, Chen P, Ma Z, Li S, Gao X, Wu R X, Pan L, Shi Y. Sensors, 2019, 19(18): 3947

[38] Arrabito G, Errico V, Zhang Z M, Han W H, Falconi C. Nano Energy, 2018, 46: 54-62

[39] Ma D, Wu X X, Wang Y G, Liao H, Wan P B, Zhang L Q. ACS Appl. Mater. Interfaces, 2019, 11(44): 41701-41709

[40] Windmiller J R, Wang J. Electroanalysis, 2013, 25(1): 29-46

[41] Zhou L, Cui Y, He C X. Chinese J. Anal. Chem., 2020, 48(4): 516-522

[42] Xiao J Y, Liu Y, Su L, Zhao D, Zhao L, Zhang X J. Anal. Chem., 2019, 91(23): 14803-14807

[43] Zhang B W, Dong Q, Korman C E, Li Z Y, Zaghloul M E. Sci. Rep., 2013, 3: 1098

[44] Zhao Z Z, Yan C, Liu Z X, Fu X L, Peng L M, Hu Y F, Zheng Z J. Adv. Mater., 2016, 28(46): 10267-10274

[45] Nie C, Frijns A, Zevenbergen M, den Toonder J. Sens. Actuators B, 2016, 227: 427-437

[46] Son D, Lee J, Qiao S, Ghaffari R, Kim J, Lee J E, Song C, Kim S J, Lee D J, Jun S W, Yang S, Park M, Shin J, Do K, Lee M,
Kang K, Hwang C S, Lu N, Hyeon T, Kim D H. Nat. Nanotechnol., 2014, 9(5): 397-404

[47] Kim J, Lee M, Shim H J, Ghaffari R, Cho H R, Son D, Jung Y H, Soh M, Choi C, Jung S, Chu K, Jeon D, Lee S T, Kim J H, Choi S H, Hyeon T, Kim D H. Nat. Commun., 2014, 5: 5747

[48] Trung T Q, Duy L T, Ramasundaram S, Lee N E. Nano Res., 2017, 10(6): 2021-2033

[49] Chen M, Ma Y J, Li Y, Wu D, Zhang Y, Youn C H. IEEE Commun. Mag., 2017, 55(1): 54-61

[50] Hao Y Q, Helo P. Robot. Comput. Integr. Manuf., 2017, 45: 168-179

[51] Qi X, Li X L, Jo H, Bhat K S, Kim S, An J, Kang J W, Lim S. Sens. Actuators A, 2020, 301: 111697

[52] Liu L, Shao J Y, Li X M, Zhao Q, Nie B B, Xu C, Ding H T. Appl. Surf. Sci., 2016, 386: 405-411

[53] Xu H, Liu J, Zhang J, Zhou G, Luo N, Zhao N. Adv. Mater., 2017, 29(31): 1700975

[54] Li B W, Yu L J, Qi J, Fu L W, Zhang P Q, Chen L X. Anal. Chem., 2017, 89(11): 5708-5713

[55] Li Z, Liu H, Ouyang C, Wee W H, Cui X, Lu T J, Pingguan-Murphy B, Li F, Xu F. Adv. Funct. Mater., 2016, 26(2): 165-180

[56] Lin D, Li B, Qi J, Ji X, Yang S, Wang W, Chen L. Sens. Actuators B, 2020, 303: 127213

[57] Qi J, Li B W, Wang X R, Zhang Z, Wang Z, Han J L, Chen L X. Sens. Actuators B, 2017, 251: 224-233

[58] Qi J, Li B, Zhou N, Wang X, Deng D, Luo L, Chen L. Biosens. Bioelectron., 2019, 142: 111533

[59] Zhou J, Li B, Qi A, Shi Y, Qi J, Xu H, Chen L. Sens. Actuators $B, 2020,305: 127462$

[60] Gong S, Schwalb W, Wang Y W, Chen Y, Tang Y, Si J, Shirinzadeh B, Cheng W L. Nat. Commun., 2014, 5: 3132

[61] Wang Z Y, Dong S, Gui M X, Asif M, Wang W, Wang F, Liu H F. Anal. Biochem., 2018, 543: 82-89

[62] Liao Q L, Mohr M, Zhang X H, Zhang Z, Zhang Y, Fecht H J. Nanoscale, 2013, 5(24): 12350-12355

[63] Xiao G, He J, Chen X, Qiao Y, Wang F, Xia Q, Yu L, Lu Z. Cellulose, 2019, 26(7): 4553-4562

[64] Yamada T, Hayamizu Y, Yamamoto Y, Yomogida Y, Izadi-Najafabadi A, Futaba D N, Hata K. Nat. Nanotechnol., 2011, 6(5): 296-301

[65] Mao Y, Ji B, Chen G, Hao C, Zhou B, Tian Y. Acs Appl. Nano Mater., 2019, 2(5): 3196-3205

[66] Ren G, Cai F, Li B, Zheng J, Xu C. Macromol. Mate Engineer, 2013, 298(5): 541-546

[67] Gong S, Cheng W. Adv Electron Mater, 2017, 3(3): 1600314

[68] Xu F, Wang X, Zhu Y, Zhu Y. Adv. Funct. Mater., 2012, 22(6): $1279-1283$

[69] Wang X D, Zhang H L, Yu R M, Dong L, Peng D F, Zhang A H, Zhang Y, Liu H, Pan C F, Wang Z L. Adv. Mater., 2015, 27(14): 2324-2331

[70] Zhao P, Deng N, Li X W, Ren C C, Wang Z Y. Sens. Actuators A, 2014, 216: $158-166$ 
[71] Stoppa M, Chiolerio A. Sensors, 2014, 14(7): 11957-11992

[72] Chow P C Y, Someya T. Adv. Mater., 2020, 32(15): 1902045

[73] Yokota T, Zalar P, Kaltenbrunner M, Jinno H, Matsuhisa N, Kitanosako H, Tachibana Y, Yukita W, Koizumi M, Someya T. Sci. Adv., 2016, 2(4): e1501856

[74] Schwarze M, Tress W, Beyer B, Gao F, Scholz R, Poelking C, Ortstein K, Guenther A A, Kasemann D, Andrienko D, Leo K. Science, 2016, 352(6292): 1446-1449

[75] Lee H, Kim E, Lee Y, Kim H, Lee J, Kim M, Yoo H J, Yoo S. Sci. Adv., 2018, 4(11): eaas9530

[76] Khan Y, Han D, Pierre A, Ting J, Wang X, Lochner C M, Bovo G, Yaacobi-Gross N, Newsome C, Wilson R, Arias A C. Proc. Natl. Acad. Sci. USA, 2018, 115(47): E11015-E11024

[77] Park S, Fukuda K, Wang M, Lee C, Yokota T, Jin H, Jinno H, Kimura H, Zalar P, Matsuhisa N, Umezu S, Bazan G C, Someya T. Adv. Mater., 2018, 30(34): 1802359

[78] Curto V F, Fay C, Coyle S, Byrne R, O'Toole C, Barry C, Hughes S, Moyna N, Diamond D, Benito-Lopez F. Sens. Actuators B, 2012, 171: 1327-1334

[79] Persano L, Dagdeviren C, Su Y W, Zhang Y H, Girardo S, Pisignano D, Huang Y G, Rogers J A. Nat. Commun., 2013, 4: 1633

[80] Gao W, Emaminejad S, Nyein H Y Y, Challa S, Chen K V, Peck A, Fahad H M, Ota H, Shiraki H, Kiriya D, Lien D H, Brooks G A, Davis R W, Javey A. Nature, 2016, 529(7587): 509-514

[81] Heikenfeld J. Electroanalysis, 2016, 28(6): 1242-1249

[82] Koh A, Kang D, Xue Y, Lee S, Pielak R M, Kim J, Hwang T, Min S, Banks A, Bastien P, Manco M C, Wang L, Ammann K R, Jang K-I, Won P, Han S, Ghaffari R, Paik U, Slepian M J, Balooch G, Huang Y, Rogers J A. Sci. Translat. Med., 2016, 8(366): 366ra165

[83] Bandodkar A J, Jeerapan I, Wang J. ACS Sens., 2016, 1(5): 464-482

[84] Zhang M, Guo S, Weller D, Hao Y, Wang X S, Ding C J, Chai K, Zou B S, Liu R B. J. Nanobiotechnol., 2019, 17: 42

[85] Matzeu G, O'Quigley C, McNamara E, Zuliani C, Fay C, Glennon T, Diamond D. Anal. Methods, 2016, 8(1): 64-71

[86] Sonner Z, Wilder E, Heikenfeld J, Kasting G, Beyette F, Swaile D, Sherman F, Joyce J, Hagen J, Kelley-Loughnane N, Naik R. Biomicrofluidics, 2015, 9(3): 031301

[87] Heikenfeld J, Jajack A, Rogers J, Gutruf P, Tian L, Pan T, Li R, Khine M, Kim J, Wang J, Kim J. Lab Chip, 2018, 18(2): $217-248$

[88] Kim J, Salvatore G A, Araki H, Chiarelli A M, Xie Z, Banks A, Sheng X, Liu Y, Lee J W, Jang K I, Heo S Y, Cho K, Luo H, Zimmerman B, Kim J, Yan L, Feng X, Xu S, Fabiani M, Gratton G, Huang Y, Paik U, Rogers J A. Sci. $A d v$., 2016, 2(8): e1600418

[89] Lochner C M, Khan Y, Pierre A, Arias A C. Nat. Commun., 2014, 5: 5745

[90] Kim T H, Lee C S, Kim S, Hur J, Lee S, Shin K W, Yoon Y Z, Choi M K, Yang J, Kim D H, Hyeon T, Park S, Hwang S. ACS
Nano, 2017, 11(6): 5992-6003

[91] Bansal A K, Hou S, Kulyk O, Bowman E M, Samuel I D W. Adv. Mater., 2015, 27(46): 7638

[92] Xu H, Yin L, Liu C, Sheng X, Zhao N. Adv. Mater., 2018, 30(33): 1800156

[93] Zang Y, Zhang F, Di C, Zhu D. Mater. Horizons, 2015, 2(2): $140-156$

[94] Pan J, Liu S Y, Zhang H Z, Lu J G. Sensors, 2019, 19(19): 4105

[95] Yang H, Qi D, Liu Z, Chandran B K, Wang T, Yu J, Chen X. Adv. Mater., 2016, 28(41): 9175

[96] Giuliani A, Placidi M, Di Francesco F, Pucci A. Reac. Funct. Polym., 2014, 76: 57-62

[97] Yang J, Wei D P, Tang L L, Song X F, Luo W, Chu J, Gao T P, Shi H F, Du C L. RSC Adv., 2015, 5(32): 25609-25615

[98] Stassi S, Cauda V, Canavese G, Pirri C F. Sensors, 2014, 14(3): 5296-5332

[99] Wang L, Li Y. Ieee Transact. Instrument. Measurement, 2013, 62(2): 495-502

[100] Segev-Bar M, Konvalina G, Haick H. Adv. Mater., 2015, 27(10): 1779

[101] Souri H, Bhattacharyya D. ACS Appl. Mater. Interfaces, 2018, 10(24): 20845-20853

[102] Wang Y, Wang L, Yang T, Li X, Zang X, Zhu M, Wang K, Wu D, Zhu H. Adv. Funct. Mater., 2014, 24(29): 4666-4670

[103] Li Y D, Nayak S, Luo Y X, Liu Y J, Mohan H, Pan J M, Liu Z J, Heng C H, Thean A V Y. Materials, 2019, 12(9): 1458

[104] Nie B Q, Li R Y, Brandt J D, Pan T R. Lab Chip, 2014, 14(6): $1107-1116$

[105] Li R Y, Nie B Q, Zhai C W, Cao J, Pan J, Chi Y W, Pan T R. Ann. Biomed. Eng., 2016, 44(7): 2282-2291

[106] Wang C, Xia K, Wang H, Liang X, Yin Z, Zhang Y. Adv. Mater., 2019, 31(9): 1801072

[107] Kaya T, Liu G, Ho J, Yelamarthi K, Miller K, Edwards J, Stannard A. Electroanalysis, 2019, 31(3): 411-421

[108] Liu Q Z, Liu Y H, Wu F Q, Cao X, Li Z, Alharbi M, Abbas A N, Amer M R, Zhou C W. ACS Nano, 2018, 12(2): 1170-1178

[109] Martín A, Kim J, Kurniawan J F, Sempionatto J R, Moreto J R, Tang G D, Campbell A S, Shin A, Lee M Y, Liu X F, Wang J. ACS Sens., 2017, 2(12): 1860-1868

[110] Lee H, Song C, Hong Y S, Kim M S, Cho H R, Kang T, Shin K, Choi S H, Hyeon T, Kim D H. Sci. Adv., 2017, 3(3): e1601314

[111] Nyein H Y Y, Bariya M, Kivimaki L, Uusitalo S, Liaw T S, Jansson E, Ahn C H, Hangasky J A, Zhao J Q, Lin Y J, Happonen T, Chao M H, Liedert C, Zhao Y B, Tai L C, Hiltunen J, Javey A. Sci. Adv., 2019, 5(8): eaaw9906

[112] Saravanakumar B, Soyoon S, Kim S J. ACS Appl. Mater. Interfaces, 2014, 6(16): 13716-13723

[113] Huang X, Liu Y, Chen K, Shin W-J, Lu C-J, Kong G-W, Patnaik D, Lee S-H, Cortes J F, Rogers J A. Small, 2014, 10(15): 3083-3090

[114] Garcia-Cordero E, Bellando F, Zhang J R, Wildhaber F, Longo $\mathrm{J}$, Guerin H, Ionescu A M. ACS Nano, 2018, 12(12): 
12646-12656

[115] Rose D P, Ratterman M E, Griffin D K, Hou L, Kelley-Loughnane N, Naik R R, Hagen J A, Papautsky I, Heikenfeld J C. IEEE Trans. Biomed. Eng., 2015, 62(6): 1457-1465

[116] Cazalé A, Sant W, Ginot F, Launay J C, Savourey G, Revol-Cavalier F, Lagarde J M, Heinry D, Launay J, Temple-Boyer P. Sens. Actuators B, 2016, 225: 1-9

[117] Matzeu G, Fay C, Vaillant A, Coyle S, Diamond D. IEEE Trans. Biomed. Eng., 2016, 63(8): 1672-1680

[118] Brueck A, Iftekhar T, Stannard A B, Yelamarthi K, Kaya T. Sensors, 2018, 18(2): 533

[119] Zheng G Z, Li K, Bu W T, Wang Y J. Build. Environ., 2019, 150: $108-118$

[120] Dey N, Ashour A S, Shi F Q, Fong S J, Sherratt R S. IEEE Trans. Consum. Electron., 2017, 63(4): 442-449

[121] Boland C S, Khan U, Backes C, O'Neill A, McCauley J, Duane
S, Shanker R, Liu Y, Jurewicz I, Dalton A B, Coleman J N. ACS Nano, 2014, 8(9): 8819-8830

[122] Atalay O, Kennon W R, Demirok E. IEEE Sens. J., 2015, 15(1): $110-122$

[123] Shaffer F, McCraty R, Zerr C L. Front. Psychol., 2014, 5: 1040-1040

[124] Pittig A, Arch J J, Lam C W R, Craske M G. Int. J. Psychophysiol., 2013, 87(1): 19-27

[125] Schwartz G, Tee B C K, Mei J G, Appleton A L, Kim D H, Wang H L, Bao Z N. Nat. Commun., 2013, 4: 1859

[126] Dias D, Silva Cunha J P. Sensors, 2018, 18(8): 2414

[127] Haahr R G, Duun S B, Toft M H, Belhage B, Larsen J, Birkelund K, Thomsen E V. IEEE Trans. Biomed. Circuits Syst., 2012, 6(1): 45-53

[128] Chacon P J, Pu L M, da Costa T H, Shin Y H, Ghomian T, Shamkhalichenar $\mathrm{H}, \mathrm{Wu} \mathrm{H}$ C, Irving B A, Choi J W. IEEE Trans. Biomed. Eng., 2019, 66(6): 1505-1513 